\title{
A Dielectric Study of Oxidation in the Amorphous and Crystalline Regions of Low Density Polyethylene with Antioxidant
}

\author{
Kazuo IIDA, Shuhei NaKamura, Masayuki IEDA, ${ }^{*}$ \\ Kazumi ITO,** and Goro SAWA \\ Department of Electronics, Mie University, Tsu 514, Japan \\ * Department of Electrical Engineering, Nagoya University, \\ Nagoya 464, Japan \\ ** Material Research and Development Department, \\ Showa Electric Wire and Cable Co., Ltd., \\ Kawasaki 210, Japan
}

(Received October 18, 1986)

\begin{abstract}
The effect of antioxidant on oxidation both in the amorphous and crystalline regions of low density polyethylene (PE) was investigated. Degradation was carried out by oxidation using thermal aging below the melting point of PE subsequent to $\gamma$-irradiation. Dielectric measurement was carried out to compare the oxidation in the amorphous region and that in the crystalline. It was found that the antioxidants had little effect on oxidation in the crystalline region, though the oxidation in the amorphous region was suppressed by antioxidant. This is interpreted in terms of dispersion of the antioxidant in PE, which is suggested by DTA study. The degree of oxidation in the crystalline region was nearly the same as that in the amorphous region if the antioxidant was doped at a higher level than a certain value. This can be interpreted as resulting from the suppression of autoxidation by virtue of the antioxidant in the amorphous region and by the insufficient supply of oxygen in the crystalline region.
\end{abstract}

KEY WORDS Polyethylene / Oxidation / Additive / Dielectric Dissipation /

Polyolefines are used as insulators in various environments because of their excellent electrical insulating properties. However, they are often degraded by heating, UV illumination and high energy irradiation. ${ }^{1-3}$ Recently, polymer insulation is demanded to sustain their electrical and mechanical properties even in severe environments. For example, electrical cables used in nuclear power generating stations are required to function in combined environments where they are exposed both to high energy irradiation and thermal aging. Therefore, insulators used under such severe conditions contain a high doping level of antioxidants. Since the mechanical degradation is usually in advance of the electrical one in such a environment of $\gamma$-irradiation and thermal aging, most of the works have been devoted to the degradation of mechanical properties. ${ }^{2}$ From the viewpoint of chemical structures, the electrical and mechanical properties are modified by crosslinking and chain scission which is accompanied with oxidation. Therefore, the infrared absorption study is powerful for obtaining information on the degradation process. $^{3,4}$ On the other hand, a dielectric study is not only important to guarantee the electrical properties directly but also powerful for elucidating the relation between degradation and morphology, since the dielectric relaxation in the amorphous region is different from that in the crystalline region. ${ }^{5}$

In semicrystalline polymers such as polyethylene, oxidation is also different between the those two regions. It is well established ${ }^{6}$ 
that oxygen is insoluble in the crystalline phase $^{7}$ so that oxidation is almost confined to the amorphous region when the polymer suffers from thermal aging and photoinitiated oxidation at temperatures below the melting point. However, when the polymer is oxidized in the molten state, existing carbonyl groups can be readily incorporated into the crystal during the crystallization process. $^{8}$ Therefore, most of the studies on dielectric relaxation in the crystalline region have been made on specimens which were oxidized in the molten state. ${ }^{5}$ In this way, the dielectric relaxation process associated with the crystalline region has been revealed, but the oxidation process in the crystal has been paid little attention. ${ }^{5}$ This is due to slight oxidation in the crystalline region and also due to the fact that the related dielectric loss is often covered with the loss which comes from ionic conduction and/or the glass-rubber relaxation in the amorphous region. ${ }^{5}$ Nevertheless, under a severe condition such as the combined radiationthermal environment, oxidation will be appreciable even in the crystal, probably because the oxygen supply is enhanced by the defect formation and the molecular motion at high temperature.

In this paper, the effect of additive on the oxidation in the amorphous and crystalline regions of $\mathrm{PE}$ is discussed mainly in relation to the dielectric results for the case of heavy doping and severe aggressive treatment. Oxidation in the crystalline region can be estimated by the dielectric dissipation factor in distinction from that in the amorphous region. It will be shown that the effect of an additive on suppression of oxidation in the crystalline region is found to be different from that in the amorphous region. This is explained in terms of dispersion of additive in PE which is detected by DTA study.

\section{EXPERIMENTAL}

The material used was low density PE (ZF30, Mitsubishi Petrochemical Co., Ltd.) having a density of $0.92 \mathrm{~g} \mathrm{~cm}^{-3}$ and crystallinity of about $50 \%$. Irganox $1010^{\circledR}$ and Antage- $F^{\circledR}$ listed in Table I were used as antioxidants. These belong to chain-breaking antioxidants which interfere with the first degradative cycle by intercepting radicals formed during chain propagation. ${ }^{1}$ The antioxidants were added in PE at the ratio shown in Table II. Film samples of $25 \mu \mathrm{m}$ nominal thickness were prepared. Such a thin film was used to avoid the influence of the oxidation process limited by diffusion of oxygen. The samples were degradated in the following way. The samples were heated at $90^{\circ} \mathrm{C}$ in air for 1 week after they had been $\gamma$-irradiated as will describe later. At temperatures higher than $90^{\circ} \mathrm{C}$ chosen here, the inflation films shrink. The $\gamma$-ray from ${ }^{60} \mathrm{Co}$ source at a dose rate of $5.0 \times 10^{4} \mathrm{rad} / \mathrm{h}$ was used for the $\gamma$-irradiation at room temperature in air and its total dose was about $20 \mathrm{Mrad}$. Dielectric measurements were carried out using a transformer bridge (1621 Precision Capacitance System; General Radio Co., Ltd.)

Table I. Description of additives

\begin{tabular}{|c|c|c|c|}
\hline Trade name & Composition & $\begin{array}{l}\text { Molecular } \\
\text { weight }\end{array}$ & $\frac{\text { Melting point }}{{ }^{\circ} \mathrm{C}}$ \\
\hline Irganox 1010 & $\begin{array}{l}\text { Pentaerythritol tetrakis }\left[3-\left(3^{\prime}, 5^{\prime}-\mathrm{di}-\right.\right. \\
\left.\text { tert-butyl- } 4^{\prime} \text {-hydroxyphenyl }\right) \\
\text { propionate] }\end{array}$ & 1178 & $110-125$ \\
\hline Antage-F & $N, N^{\prime}$-2-Naphthyl $p$-phenylenediamine & 360.46 & $>225$ \\
\hline
\end{tabular}


Table II. Sample description

\begin{tabular}{|c|c|c|}
\hline \multirow{2}{*}{ Sample } & \multirow{2}{*}{ Additive } & $\begin{array}{l}\text { Additive } \\
\text { content }\end{array}$ \\
\hline & & $w t \%$ \\
\hline B & & 0 \\
\hline IR-5 & Irganox 1010 & 5 \\
\hline----1 & ------ & ---- \\
\hline AF-5 & Antage-F & 5 \\
\hline$A F-10$ & & 10 \\
\hline
\end{tabular}

over a temperature range from $-100^{\circ} \mathrm{C}$ to $100^{\circ} \mathrm{C}$.

\section{RESULTS AND DISCUSSION}

Change of infrared spectra brought by the $\gamma$ irradiation and the subsequent heat treatment is shown in Figure 1. A carbonyl $\mathrm{C}=\mathrm{O}$ band at $1715 \mathrm{~cm}^{-1}$ appears in the spectra of the sample $\mathrm{B}$ after treatment, as shown in Figure 1(a). It can be seen that the sample B was heavily oxidized by the treatment because of no antioxidant in the sample. As Irganox 1010 contains carbonyl groups as shown in Table I, an absorption peak at $1740 \mathrm{~cm}^{-1}$ was found in the sample IR-5 even without the treatment as shown in Figure 1(b). Other absorption peaks due to Irganox 1010 appeared in the sample IR-5 at $3650 \mathrm{~cm}^{-1}$ and at $1150-1250 \mathrm{~cm}^{-1}$, which were attributed to $\mathrm{OH}$-stretching and $\mathrm{C}\left(\mathrm{CH}_{3}\right)_{3}$, respectively. The additional carbonyl absorption peak resulting from oxidation in the sample IR-5 appeared after the treatment, which was smaller than that of the sample B. For the sample AF-5, absorption peaks due to Antage-F appeared at $3380 \mathrm{~cm}^{-1}$ caused by NH-stretching and 1600-1630 $\mathrm{cm}^{-1}$ by naphthalene rings, as shown in Figure 1(c). As the sample AF-5 was treated, the carbonyl absorption peak appeared, which was also smaller than that for the sample B. From the above IR results, it is clear that doping of the antioxidants is effective for suppression of oxidation.

Figure 2 shows the temperature dependence of $\tan \delta$ for nontreated samples B, IR-5 and AF-5. In the sample IR-5, $\tan \delta$ shows a large peak at about $80^{\circ} \mathrm{C}$. Irganox 1010 , which is doped in the sample IR-5, contains polar groups. The molecular motion of the polar groups combined with the benzene ring will be released at a higher temperature than the transition temperature of PE. Therefore, the $\tan \delta$ peak around $80^{\circ} \mathrm{C}$ for the sample IR-5 is probably due to the carbonyl groups in Irganox 1010. Except for the $\tan \delta$ peak due to Irganox 1010, the additives used have little influence on $\tan \delta$, while $\tan \delta$ increased slightly with increasing its content over the whole temperature range studied.

Figure 3 shows the temperature dependence of $\tan \delta$ for the $\gamma$-irradiated and heat treated samples. From a comparison between Figures 2 and 3 , it is definite that the treatment brings larger $\tan \delta$. The increase in $\tan \delta$ is caused by polar groups of oxidation products. For the sample B with treatment, three peaks appeared at about $-100^{\circ} \mathrm{C}$, room temperature and about $55^{\circ} \mathrm{C}$, respectively, at $1 \mathrm{kHz}$. These $\tan \delta$ peaks are labeled here for convenience sake as $\alpha, \beta$, and $\gamma$, respectively, in order of descending temperature, as shown in Figure 3. Judging from the peak temperatures, the $\alpha$ peak is attributed to the crystalline region, the $\beta$ peak comes from the segmental motion of chain molecules in the amorphous region of $\mathrm{PE}$ and the $\gamma$ peak is ascribed to the local motion of the molecules. ${ }^{5,9,10}$ In Figure 3, it is definite that the $\beta$ and $\gamma$ peaks in the samples IR-5 and AF5 are smaller than those in the sample $B$. This fact indicates that the additive has an effect on suppression of oxidation in the amorphous region of $\mathrm{PE}$.

The $\beta$ peak in the sample $\mathrm{B}$ is so large that the $\alpha$ peak appears as a shoulder of the $\beta$ peak. Therefore, an accurate comparison of the $\alpha$ peak in the sample B with those of samples IR5 and AF-5 is difficult and so we will try to separate the $\beta$ peak from the observed curve. 


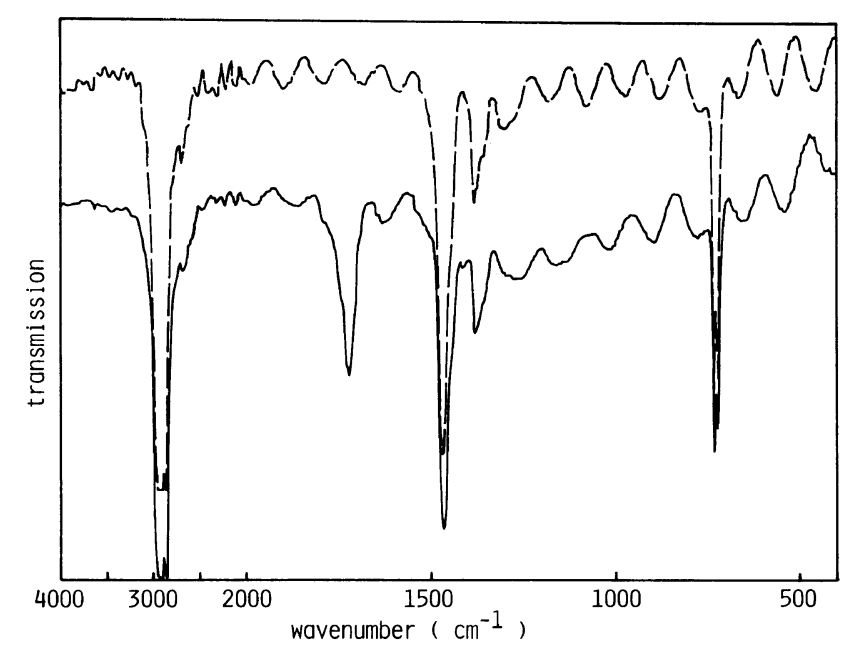

(a)

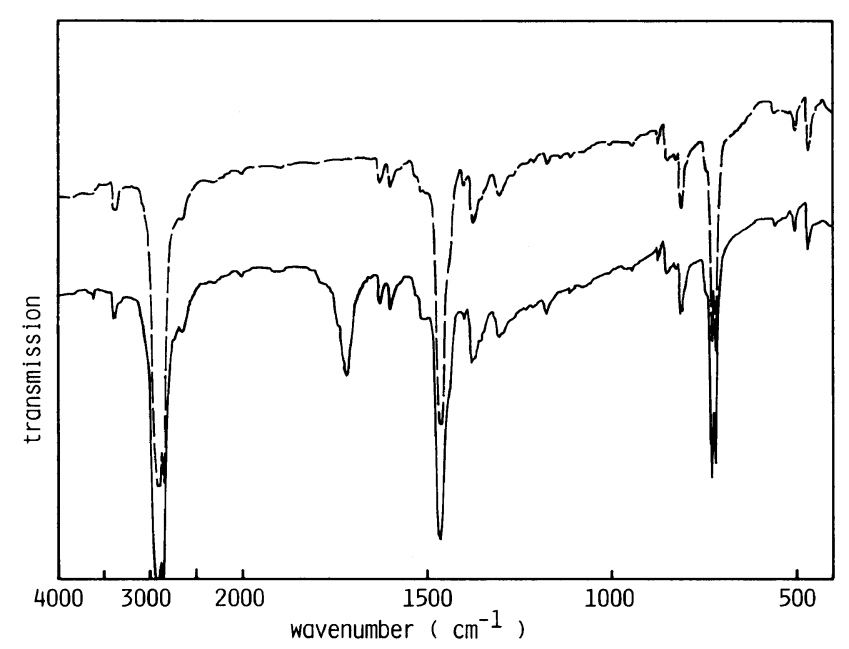

(b)

Empirically, dielectric relaxation is described by the Cole-Cole equation ${ }^{11}$;

$$
\varepsilon_{\mathrm{r}}=\varepsilon_{\mathrm{r} \infty}+\left(\varepsilon_{\mathrm{r} 0}-\varepsilon_{\mathrm{r} \infty}\right) \frac{1}{1+\left(i \omega \tau_{0}\right)^{1-\alpha}}
$$

where $\varepsilon_{\mathrm{r}}$ is the complex dielectric constant, $\varepsilon_{\mathrm{r} 0}$ and $\varepsilon_{\mathrm{r} \infty}$ the limiting values of dielectric constant at very low and high frequencies, $\omega$ the angular frequency, $\tau_{0}$ the dielectric relaxation time and $\alpha$ the distribution parameter in the Cole-Cole equation. Separation of real and imaginary parts of eq 1 gives

$$
\varepsilon_{\mathrm{r}}^{\prime}=\varepsilon_{\mathrm{r} \infty}+\frac{1}{2}\left(\varepsilon_{\mathrm{r} 0}-\varepsilon_{\mathrm{r} \infty}\right)
$$

$$
\begin{gathered}
\times\left[1-\frac{\sinh \left\{(1-\alpha) \ln \omega \tau_{0}\right\}}{\cosh \left\{(1-\alpha) \ln \omega \tau_{0}\right\}+\sin \frac{1}{2} \alpha \pi}\right] \\
\varepsilon_{\mathrm{r}}^{\prime \prime}=\frac{1}{2}\left(\varepsilon_{\mathrm{r} 0}-\varepsilon_{\mathrm{r} \infty}\right) \frac{\cos \frac{1}{2} \alpha \pi}{\cosh \left\{(1-\alpha) \ln \omega \tau_{0}\right\}+\sin \frac{1}{2} \alpha \pi}
\end{gathered}
$$




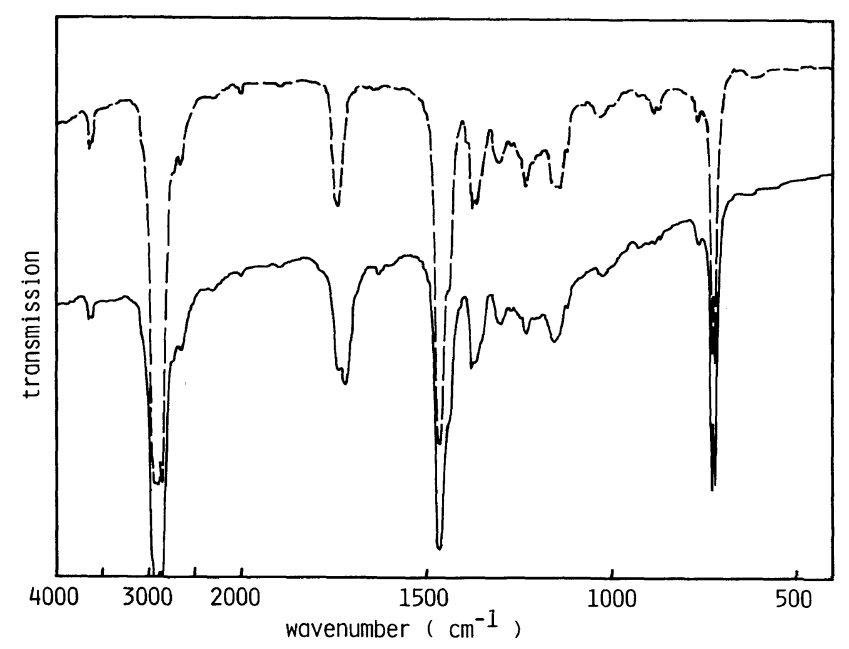

(c)

Figure 1. Infrared spectra of samples B(a), IR-5(b), and AF-5(c). Solid lines indicate samples subjected to $\gamma$-irradiation and subsequent heat treatment. Dashed lines represent untreated samples.

The relaxation time of segmental motion is usually represented by WLF equation. However, the relation between $\log f_{\mathrm{m}}$ and $1 / T$ for semicrystalline polymers is often linear at high temperatures. ${ }^{5,9}$ Therefore, it is assumed as a rough approximation that the relaxation follows the Arrhenius equation:

$$
\tau_{0}=A \exp \left(E_{\mathrm{a}} / k T\right)
$$

where $A$ and $E_{\mathrm{a}}$ are constants, $k$ the Boltzmann's constant and $T$ the temperature. For the case that parameters $\alpha, A$, and $E_{\mathrm{a}}$ in eq $1-4$ are constant over the whole temperature range of interest, the following expressions for the temperature variation of $\varepsilon_{\mathrm{r}}^{\prime}$ and $\varepsilon_{\mathrm{r}}^{\prime \prime}$ are obtained:

$$
\begin{aligned}
\varepsilon_{\mathrm{r}}^{\prime} & =\varepsilon_{\mathrm{r} \infty}+\frac{1}{2}\left(\varepsilon_{\mathrm{r} 0}-\varepsilon_{\mathrm{r} \infty}\right) \\
& \times\left[1-\frac{\sinh \left\{(1-\alpha)\left(\frac{E_{\mathrm{a}}}{k T}+\ln A \omega\right)\right\}}{\cosh \left\{(1-\alpha)\left(\frac{E_{\mathrm{a}}}{k T}+\ln A \omega\right)\right\}+\sin \frac{1}{2} \alpha \pi}\right]
\end{aligned}
$$

(5) With the help of eq $7, \ln A \omega$ in eq 5 and 6 can be expressed in terms of $k, E_{\mathrm{a}}$, and $T_{\mathrm{m}}$, and we

$$
\begin{aligned}
\varepsilon_{\mathrm{r}}^{\prime \prime}= & \frac{1}{2}\left(\varepsilon_{\mathrm{r} 0}-\varepsilon_{\mathrm{r} \infty 0}\right) \\
& \times \frac{\cos \frac{1}{2} \alpha \pi}{\cosh \left\{(1-\alpha)\left(\frac{E_{\mathrm{a}}}{k T}+\ln A \omega\right)\right\}+\sin \frac{1}{2} \alpha \pi}
\end{aligned}
$$

In addition to the explicit temperature dependent terms, eq 5 and 6 include the implicit temperature dependent terms via $\varepsilon_{\mathrm{r} 0}$ and $\varepsilon_{\mathrm{r} \infty}$. Since $\varepsilon_{\mathrm{r} \infty}$ varies only with density, the temperature dependence of $\varepsilon_{\mathrm{r} 0}$ is negligible in the present case. The temperature dependence of $\varepsilon_{\mathrm{r} 0}$ is appreciable, but small as compared with the variation of the hyperbolic functions in eq 5 and 6 .

It follows from eq 6 that $\varepsilon_{\mathrm{r}}^{\prime \prime}$ at a fixed frequency $\omega$ has a maximum at a temperature $T_{\mathrm{m}}$ where the argument of the hyperbolic cosine in eq 6 is zero. Then, we obtain

$$
1 / T_{\mathrm{m}}=-\left(k / E_{\mathrm{a}}\right) \ln A \omega
$$
,

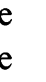




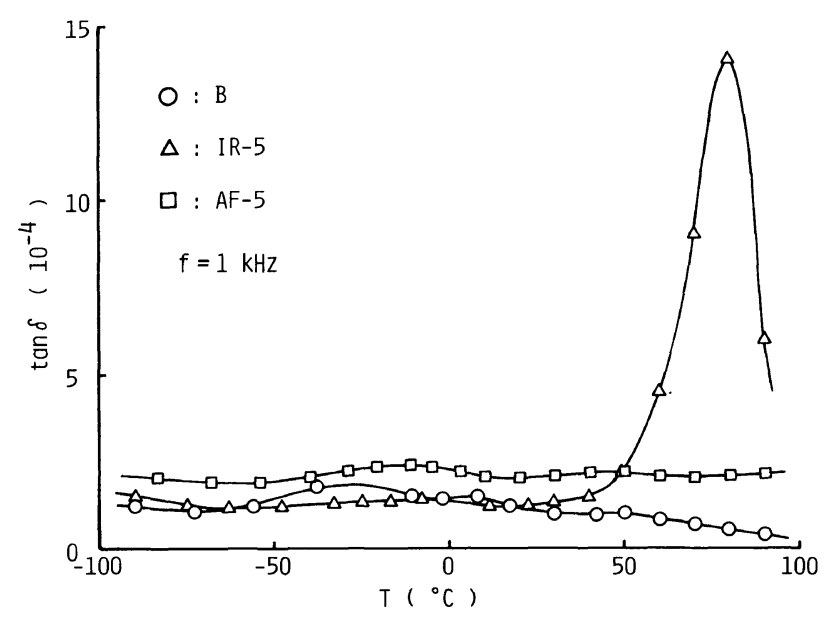

Figure 2. Temperature dependence of $\tan \delta$ for untreated samples B, IR-5, and AF-5.

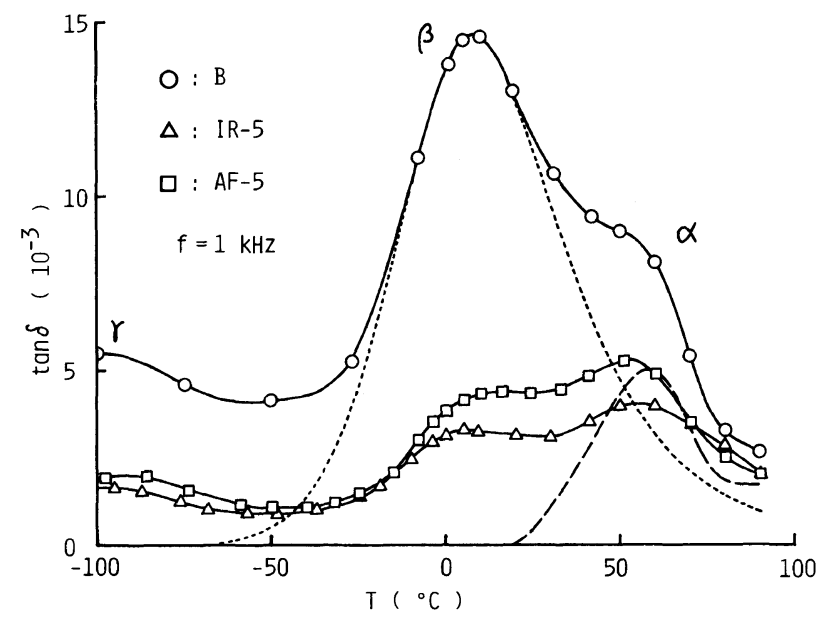

Figure 3. Temperature dependence of $\tan \delta$ for samples, B, IR-5, and AF-5 treated with the $\gamma$-irradiation and subsequent heat treatment.

obtain

$$
\begin{aligned}
\varepsilon_{\mathrm{r}}^{\prime} & =\varepsilon_{\mathrm{r} \infty}+\frac{1}{2}\left(\varepsilon_{\mathrm{r} 0}-\varepsilon_{\mathrm{r} \infty 0}\right) \\
& \times\left[1-\frac{\sinh \left\{\frac{(1-\alpha) E_{\mathrm{a}}}{k}\left(\frac{1}{T}-\frac{1}{T_{\mathrm{m}}}\right)\right\}}{\cosh \left\{\frac{(1-\alpha) E_{\mathrm{a}}}{k}\left(\frac{1}{T}-\frac{1}{T_{\mathrm{m}}}\right)\right\}+\sin \frac{1}{2} \alpha \pi}\right]
\end{aligned}
$$

(8) of $\varepsilon_{\mathrm{r} 0}$ and $\varepsilon_{\mathrm{r} \infty}$ is neglected, the temperature dependence of

$$
\tan \delta=\varepsilon_{\mathrm{r}}^{\prime \prime} / \varepsilon_{\mathrm{r}}^{\prime}
$$




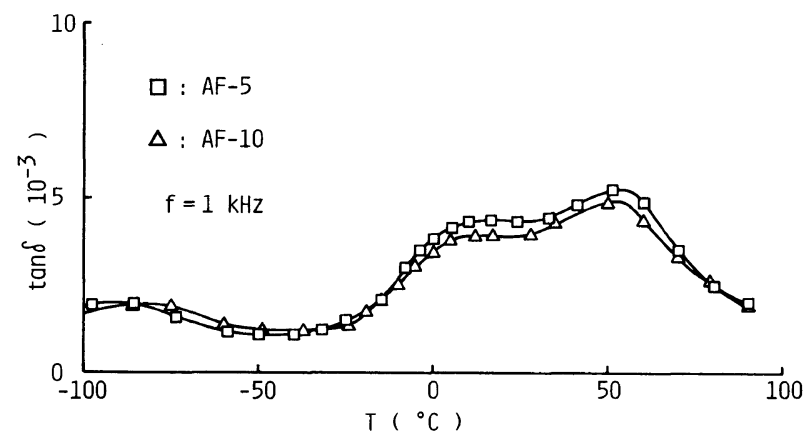

Figure 4. Temperature dependence of $\tan \delta$ for samples AF-5 and AF-10 with the $\gamma$-irradiation and subsequent heat treatment.

is calculated by using eq 8 and 9 .

The dotted line in Figure 3 represents the calculated values from eq $8-10$ for the parameters: $\varepsilon_{\mathrm{r} 0}=3.11, \varepsilon_{\mathrm{r} \infty}=2.56, T_{\mathrm{m}}=10^{\circ} \mathrm{C}$, $E_{\mathrm{a}}=2.3 \mathrm{eV}$, and $\alpha=0.81$. The parameters were determined by the following procedure. At first, the approximate values of $\varepsilon_{\mathrm{r} 0}$ and $\varepsilon_{\mathrm{r} \infty}, T_{\mathrm{m}}$ and $E_{\mathrm{a}}$ were estimated from the $\varepsilon_{\mathrm{r}}^{\prime}-T$, $\varepsilon_{\mathrm{r}}^{\prime \prime}-T$, and $f_{\mathrm{m}}-1 / T$ curves, respectively. Finally, the values of $\varepsilon_{\mathrm{r} 0}, \varepsilon_{\mathrm{r} \infty}, T_{\mathrm{m}}, E_{\mathrm{a}}$, and $\alpha$ were determined in such a way as to give a good fit to the $\tan \delta-T$ curve. The net $\alpha$ peak in the sample B can be represented by a dotted line which is obtained by subtracting the dotted line from the corresponding solid line. The results are shown by the broken line in Figure 3. The net $\alpha$ peak thus estimated for the sample B has roughly the same magnitude as the $\alpha$ peaks for the sample IR- 5 and AF-5. It thus follows that the antioxidants used seem to have little effect on the suppression of oxidation which is responsible for the $\alpha$ peak. Since the $\alpha$ peak is known to be related to the crystalline region, ${ }^{5,9,10}$ it can be concluded that the oxidation in the crystalline region can be hardly prevented by doping the antioxidants used. Figure 4 shows the case of much heavier doping (AF-10) of Antage-F to compare with the sample AF-5. This result indicates that no distinctive effect of higher additive content than the present one can be expected to pre-

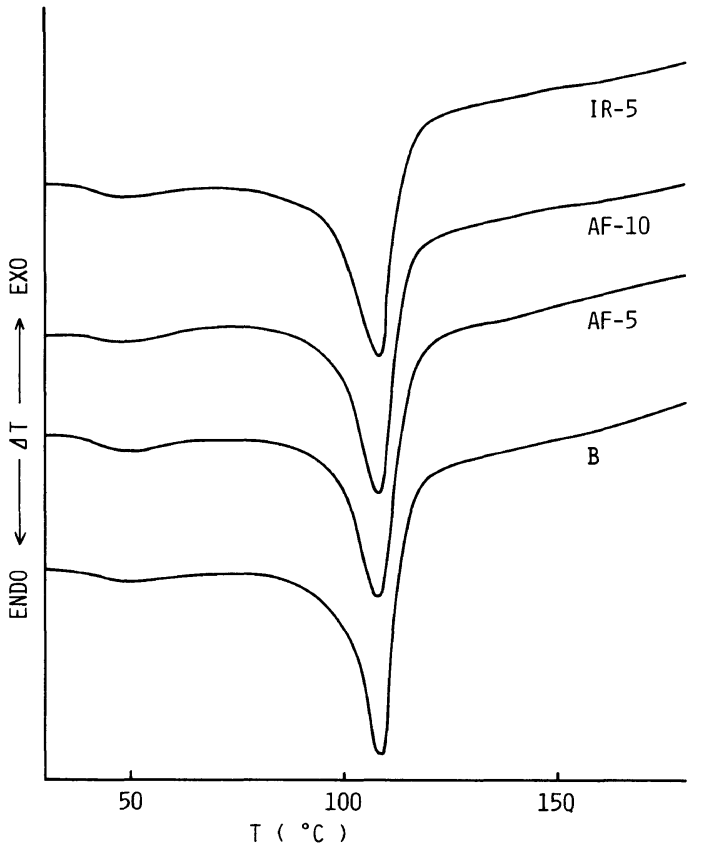

Figure 5. The heating DTA curves for the melting of crystals of untreated samples.

vent the oxidation.

There are two possible reasons for the little effect of antioxidants on the suppression of oxidation in the crystalline region: one is the modification of crystalline size by doping, enhancing the oxygen supply; the other is that an antioxidant molecule hardly approaches the interface between the amorphous and crystalline regions so that the function of the anti- 
oxidant is of little use. To examine the change in crystalline size with doping, DTA measurements were made at a heating rate of $10 \mathrm{~K} \mathrm{~min}^{-1}$ in air. The results are shown in Figure 5. The heating DTA curves exhibit an endothermic peak with the maximum at $109^{\circ} \mathrm{C}$. This peak is associated with the melt of the crystal of PE. No change of the peak temperature and area can be seen with doping of Irganox 1010 and Antage-F to PE. This means that the doping induces no change both in size and quantity of the crystal of PE. It can be said that the antioxidants affect scarcely the crystal and exist seldomly near and inside the crystalline region, but mostly in the amorphous region. This might lead to the conclusion that the little effect of antioxidant on the suppression of oxidation in the crystalline region is ascribed to the absence of the additive in this region.

In the oxidized samples IR-5, AF-5, and AF-10, the $\alpha$ peak has the same order of magnitude as the $\beta$ peak. Supposing the maximum value of $\tan \delta$ is a measure of the number of oxidation products, the degree of oxidation in the amorphous and crystalline regions of these samples might be nearly the same, since the crystallinity is about $50 \%$. This result can be interpreted as follows. Free radicals produced by $\gamma$-irradiation combine with oxygen, yielding the carbonyl goups through hydroperoxides. The autoxidation ${ }^{1}$ is prevented by the antioxidant if the doping is higher than a certain level in the amorphous region and by lack of oxygen supply in the crystalline region. However, since the antioxidant reacts with alkyl-peroxy radical rather than alkyl radical because of rapid reaction between alkyl radical and oxygen, the concentration of carbonyl groups would be controlled mainly by primary free radicals produced by the $\gamma$-irradiation. Therefore, the presence of antioxidants cannot reduce the present level of oxidation, but will be effective in preventing autoxidation at a higher dose of $\gamma$-rays.

\section{CONCLUSIONS}

The effects of antioxidant on the oxidation of low density polyethylene was investigated after heavy doping and severe treatment. As generally accepted, the antioxidants were found to be effective in the suppression of oxidizing PE in the general sense from IR data. To discuss the functions of the antioxidants in the amorphous and crystalline regions separately, a dielectric study was made. It is concluded from the dielectric results that the antioxidants had little effect on the oxidation in crystalline region of PE, although the oxidation in the amorphous region of PE was suppressed by antioxidants. From a DTA study, it was found that the antioxidants were absent near and within the crystalline region. It was also found that at a higher doping level than a certain value, the amorphous region had nearly the same concentration of carbonyl groups as the crystalline region. This can be interpreted to be due to the prevention of autoxidation by the presence of antioxidants in the amorphous region and by lack of oxygen in the crystalline region.

Acknowledgments. The authors would like to thank Messrs. H. Abe, T. Itoh, K. Ishida, and M. Miwa for their helpful assistance in carrying out the experiments.

\section{REFERENCES}

1. N. S. Allen, Ed., "Degradation and Stabilisation of Polymers," Applied Science Publishers, London and New York, 1983.

2. C. D. Bopp and O. Sisman, Nuclenic, 13, No. 10, 51 (1955).

3. D. J. Carlsson and M. D. Wiles, Macromolecules, 2, 587 (1969).

4. ZS. Fodor, M. Iring, F. Tüdos, and T. Kelen, $J$. Polylm. Sci., Polym. Chem. Ed., 22, 2539 (1984).

5. C. R. Ashcraft and R. H. Boyd, J. Polym. Sci., Polym. Phys. Ed., 14, 2153 (1976).

6. N. C. Billingham and P. D. Calvert, Dev. Polym. Stab., 3, 139 (1980). 
A Dielectric Study of Oxidation in LDPE

7. A. S. Michaels and H. J. Bixler, J. Polym. Sci., 50, 10. Y. Wada and K. Tsuge, Jpn. J. Appl. Phys., 1, 64 393, 413 (1961). (1962).

8. D. Bodily and B. Wunderlich, J. Polym. Sci., A-2, 4, 11. C. J. F. Bottcher and P. Bordewijk, "Theory of 25 (1966).

9. Y. Wada, J. Phys. Soc. Jpn., 16, 1226 (1961). Electrical Polarization," Elsevier Scientific Publishing Co., New York, 1978, Chapter 9. 